\title{
EFFETS DU MODE DE CONSERVATION D'UN MAÏS IMMATURE SUR L'UTILISATION DE SES GLUCIDES ET DE SON AZOTE CHEZ LE PORC EN CROISSANCE-FINITION
}

\author{
L. P. BORGIDA, J. DELORT-LAVAL et G. VIROBEN \\ avec la collaboration technique de Monique Diez, \\ Michèle Fiszlewicz, Françoise Kozlowski et S. Gueneau \\ Laboratoire de Technologie des Aliments des Animaux, \\ Centre national de Recherches zootechniques, I. N. R. A., \\ r8350 Jouy en Josas
}

\section{RÉSUMÉ}

La conservation de l'épi enticr de maïs immature (50 p. Ioo de matière sèche) par déshydratation $\left(\mathrm{I} 3 \mathrm{O}^{\circ} \mathrm{C}, \mathrm{I} h\right)$ provoque une réduction notable de son taux d'azote soluble en milieu trichloracétique à to $\mathrm{p}$. Ioo $(\mathrm{p} / \mathrm{v})$ et l'accroissement de la sensibilité de son amidon à l' $\alpha$-amylase. Son ensilage entraîne une augmentation des taux d'azote non protéique et ammoniacal, une réduction du taux de lysine ( -30 p. Ioo) et des glucides solubles, avec formation d'acide lactique et d'acides gras volatils.

Le porc mâle castré en finition digère mieux les composants du maïs ensilé distribué seul, dont la valeur énergétique nette est de $0,88 \mathrm{UF} / \mathrm{kg}$ MS et l'utilisation digestive de l'azote de $7 \mathrm{I}$ p. Ioo contre respectivement $0,8 \mathrm{I} \mathrm{UF} / \mathrm{kg}$ MS et $56 \mathrm{p}$. Ioo pour le déshydraté. Un régime équilibré par un apport azoté de bonne qualité ne procure qu'une amélioration limitée de la rétention azotée à partir d'ensilage : 36,9 p. roo contre 52,9 avec l'épi déshydraté.

L'amidon et les glucides solubles de l'ensilage sont, sous l'effet d'une forte activité amylasique $(25 \mathrm{U} / \mathrm{ml})$, davantage dégradés dans l'estomac, alors que c'est surtout dans l'intestin grêle (300 à I $400 \mathrm{U} / \mathrm{ml}$ ) et le cæcum qu'est digéré l'amidon de l'épi séché. L'aliment et la phase liquide des contenus digestifs transitent plus rapidement. La dégradation plus lente des glucides et leur absorption dans les parties distales du tube digestif pourraient être en relation avec la meilleure rétention de l'azote du régime à base d'épi déshydraté.

\section{INTRODUCTION}

Pour conserver l'épi de maïs immature, la déshydratation est une technique de choix ; mais l'ensilage offre l'avantage d'une mise en œuvre peu côtteuse et présenterait un intérêt économique certain dans la mesure où les fermentations qư il induit n'altèrent pas l'efficacité nutritive de la céréale. 
Chez le porc, peu de travaux concernent ces produits de conservation ; ColomBUS, HARNISCH et JAHN (I957), étudiant la valeur alimentaire des différentes parties du mais vert ou conservé par ensilage, notent que l'ensilage d'épis entiers avec spathes (40 p. Ioo de matière sèche - MS) améliore la digestibilité de l'azote, et diminue celle des autres composants de la céréale. Gross (I970) montre l'effet défavorable de l'état de maturité du grain sur la digestibilité de l'azote et de la cellulose de l'épi de maiss. Sur des grains récoltés à $60 \mathrm{p}$. Ioo MS, le même auteur met en évidence une diminution de digestibilité de la matière organique en fonction de son mode de conservation (88,4 p. Ioo en vert, 86, I ensilé et 85,2 séché). De leur côté, HolmEs, BAyLEY et HorNEY (I973) notent également la plus faible digestibilité de la MS des rations à base de maîs immature séché $(82,9$ p. Ioo) par rapport à l'ensilé $(86, \mathrm{I})$ chez le porc en croissance, alors que, dans le cas du blé et de l'orge, HellbERG (I963) ne trouve que des différences minimes.

Du fait de sa forte humidité initiale, le maïs immature est, au cours du séchage soumis à un traitement thermique intense, susceptible d'agir sur sa digestion et son efficacité métabolique. La conservation par fermentation transforme notablement, quant à elle, les fractions glucidique et azotée solubles de la céréale ; c'est pourquoi il nous a paru intéressant de comparer les effets de ces deux traitements sur la composition et les caractéristiques physico-chimiques de l'épi de maïs immature, sur les modalités de sa digestion et sur son efficacité digestive et métabolique chez le porc en croissance. Cette étude fait suite à des essais de conservation d'orge et de maîs immatures (GOUET, RIOU et BOUSSET-FATIANOFF, I97I) testés par ailleurs sur le taurillon (ZELTER, Charlet-Lery et Tisserand, I97I).

\section{MATÉRIEI, ET MÉTHODES}

\section{A. - Technologie et composition du maïs immature}

\section{Traitement de la cévéale.}

L'épi de maïs (variété INRA 260) est récolté à environ 50 p. Ioo d'humidité avec un corn picker réglé pour n'éliminer qu'un minimum despathes. Il est haché à poste fixe dans une ensileuse à couteaux, puis dans un broyeur à fourrage. Une partie est ensilée dans deux silos-cuve en béton d'un $\mathrm{m}^{3}$ de capacité, $1^{\prime}$ autre séché durant I heure dans un conrant d'air chauffé à $\mathrm{I} 25^{-\mathrm{I}} 35^{\circ} \mathrm{C}$, puis divisée dans un broyeur à marteaux.

Avant đistribution aux animaux, l'ensilage est dilacéré cians un broyeur à marteau sans grille, de manière à obtenir des particules de taille réduite et plus homogène.

\section{Caractérisation des produits.}

La composition du maïs est déterminée par les méthodes officielles d'analyse. Sur un échantillon prélevé après ensilage ou déshydratation et broyé dans l'azoto liquide, un fractionnement azoté est effectué selon la technique de Baudet et al. (1966). Sa solubilité en milieu trichloracétique à Io $\mathrm{p}$. Ioo $(\mathrm{p} / \mathrm{v})$ définit l'azote non protéique. L'azote ammoniacal est dosé par microdiffusion (CONWAY, I957). Les acides aminés libres sont fixés par passage de l'extrait aqueux à $0^{\circ} \mathrm{C}$ sur colonne d'Amberlite IR Izo et éluées par des concentrations successives d'ammoniaque $2 \mathbb{N}$ puis $4 \mathrm{~N}$. La libération des acides aminés des échantillons initiaux ou de leur fraction non protéique est obtenue par hydrolyse dans l'acide $6 \mathrm{~N}$ à $\mathrm{r} 30^{\circ} \mathrm{C}$ pendant 24 heures. Leur séparation et leur dosage sont effectués à l'aide d'un analyseur automatique (Moore. Spackmann et Stein, 1958).

Les glucides solubles dans l'alcool $80^{\circ} \mathrm{GL}$ et ceux extraits à l'alcool $40^{\circ} \mathrm{GL}$ et précipitables à l'iode (HASSID et NEUFELD, 1968) sont dosés par la méthode à l'orcinol sulfurique de Tillmans et Philippi adaptée au dosage automatique par Kesler (I967). L'amidon est déterminé par la 
méthode enzymatique de ThIVENi, Mercier et (iuilbot (i965) et soumis à un test d'hydrolyse par l' $\alpha$-amylase bactéricnne de $B$. subtilis (Mercier, 1968).

Les acides gras volatils (acétique, propionique, butyrique), sont déterminés dans l'ensilage par chromatographie de partage gaz liquide selon JAMks et MAKTIN modifiée par ZELTER et LEROY (1958) et l'acide lactique sclon BARNETT (I9.5I).

\section{B.- Bilans sur porc en croissance-finition}

Des bilans d'azote et de matière organique sont établis sur cles porcs en croissance recevant la céréalc soit seule, soit supplémentée par du tourteau de soja.

\section{Animaux.}

Huit porcs mâles castrés de race Large White provenant de trois portées contemporaines, sont répartis en deux lots en fonction de leur poids initial $(52-65 \mathrm{~kg})$.

\section{Régimes et mode d'alimentation.}

Les régimes sont composés de maïs immature cnsilé ou déshydraté et d'un composé minéral (1) distribué à raison de $I$ p. I oo de la matière sèche du maïs. La méthionine est l'acide aminé limitant de l'efficacité d'un régime maïs-soja; nous avons donc ajouté un supplément de cet acide a miné pour atteindre un taux calculé de ${ }_{4}, 2$ p. Ioo des matières azotées du régime.

La ration journalière est distribuée en deux repas quotidiens égaux, à raison de $30 \mathrm{~g}$ de matière sèche par $\mathrm{kg}$ de poids vif pour les céréales et de $5 \mathrm{~g}$ pour le complément azoté. Pour l'ensilage, après élimination de la conche supérieure du silo, les rations, pesées deux fois par semaine, sont conservées au réfrigérateur à $+4^{\circ} \mathrm{C}$ à l'abri de l'air. Dans tous les régimes, la teneur en matière sèche de's repas est ajustéc au voisinage de $30 \mathrm{p}$. 100.

\section{Collecte des échantillons.}

Le contrôle des quantités ingérées est réalisé par pesée des aliments distribués et des refus. Les échantillons destinés à l'analyse sont séchés à l'étuve infrarouge à soo C et broyés.

Les fèces, préalablement pulvérisées d'acide sulfurique à $25 \mathrm{p}$. $100(\mathrm{p} / \mathrm{p})$, sont prélevés quotidiennement, homogénéisés au broyeur et échantillonnés proportionnellement ( Io p. Ioo) à leur poids frais. Une détermination de matière sèche est effectuée sur roo g de fèces frais.

L'urine est recueillie dans $20 \mathrm{ml}$ d'acide sulfurique à $25 \mathrm{p}$. Ioo (p/p), son poids et sa densité sont déterminés; un prélèvement aliquote quotidien est cumulé et conservé au froid.

\section{Dispositif expérimental.}

Au début de l'essai, les animaux sont répartis en 4 groupes. lls reçoivent la céréale ensilée ou déshydratée, soit seule, soit supplémentéc par le tourteau de soja. La mesure du bilan est effectuée sur deux périodes successives, chacune de huit jours, précédéc d'un temps d'adaptation minimum de dix jours. Dans la seconde période, chaque groupe change à la fois de régime de base et de taux azoté. Les animaux sont placés en cages à métabolismeindividuclles (FArRIES ct OSLAGE, I96I)et pesés deux jours consécutifs avant et après la période de récolte.

\section{C. - Prélèvoments digestifs après abattage}

\section{Aliments et animaux.}

Cinq porcs d'un poids vif moyen de $60 \mathrm{~kg}$ reçoivent durant dix-huit jours un régime à I Io g de matières azotées digestibles par unité fourragère, comprenant le maïs immature ensilé ou déshydraté, une farine de hareng de Norvège, à raison de 8 p. roo de la matière sèche distribuée, et I p. Ioo de complément minéral ( $\left.{ }^{1}\right)$; io g de polyéthylèneglycol 4 ooo (PEG) sont ajoutés au repas test qui précède l'abattage.

(1) Composition centésimale du composé minéral : carbonate de $\mathrm{Ca} 9,49$; phosphate bicalcique 55,0; sel marin 30,0; sulfate de Cu 0,06 ; de Zn I,5; de Co o,or; de Mn 0,6 ; de Fe 3, o et de sodium 0,34 . 


\section{Collecte dés échantillons.}

Les animaux sont sacrifiés à divers intervalles (30-60-180 minutcs) après la fin de ce repas d'une durée d'une heure. Les contenus des différentes parties du tube digestif (estomac, intestin grêle, cxcum, gros intestin) sont prélevés, pesés ct homogénéisés. Sur des parties aliquotes sont effectués le dosage direct du PIEG et, après dessication à l'étuve infrarouge à 8oC, l'analyse cles composants majeurs. Le fractionnement des glucides et la détermination de l'activité amylasique portent sur un échantillon immédiatement plongé dans un bain d'alcool froid et conservé à - I $80^{\circ}$.

\section{Méthodes analytiques.}

Le PEG est closé par la méthode de Sмгтн (1959). Les méthodes appliquées aux composants majeurs et aux glucides des contenus intestinaux sont celles décrites pour les aliments. Après déprotéinisation de l'extrait, les glucides de la fraction soluble clans l'alcool $80^{\circ} \mathrm{GL}$ sont identifiés par chromatographie unidimensionnelle sur couche mince de gel de silice G. La séparation en est effectuée sous l'action successive d'un mélange propanol/acétate d'éthyle/eau — I f/2/7 (CommERFORD, VAN DUZEE et SCALlET, I 963) durant I 6 heures, puis d'un mélange méthanol/acétate d'éthyle/eau-36/52/1 2 (Huber, Scobel et HAx TAI, 1966) durant 5 heures. La révélation est faite à l'aide du réactif de Giri et Nigam (I953).

L'activité amylasique est déterminée, sur la phase liquide des contenus intestinaux séparés par centrifugation à froid, selon une technique dérivée de celle de Somogyr (Anonyme, i968).

\section{RÉSUL'TA'TS}

\section{I. - Caractérisation de l'épi de céréale}

La composition moyenne des aliments de cette expérience est donnée dans le tableau $\mathrm{r}$.

TABLEAU I

Composition brute moyenne des aliments expérimentaux (p. Ioo MS)

Average crude composition of experimental feeds (p. roo DM)

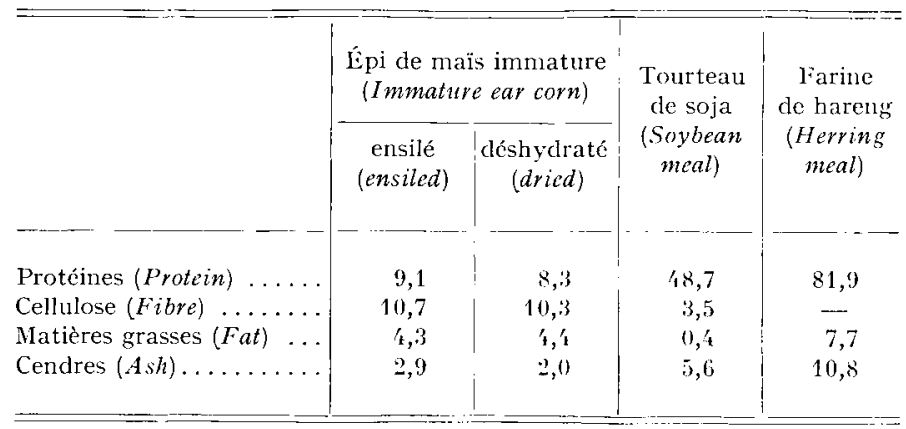

L'ensilage d'épi de mais a une teneur moyenne en matière sèche de 53,5 p. Ioo durant la première période, de $56,0 \mathrm{p}$. roo durant la seconde. Il n'a pas été tenu compte, dans les calculs, de la correction de matière sèche proposée par FATIANOFF et Gouer (I969), qui joue peu dans le cas des ensilages de maîs et devrait englober d'autres substances volatiles (alcools) non dosées. On retrouve, au désilage, en pourcentage du produit tel quel, 0,675 d'acide acétique, 0,028 de propionique, une faible 
teneur en $\mathrm{N}$ ammoniacal (0,029 p. I00) et un taux élevé (I,32 p. I00) d'acide lactique, caractéristiques d'un ensilage de bonne qualité. L'activité amylasique du produit est de $22 \mathrm{U} / \mathrm{g}$ MS.

\section{a) Matières azotées.}

Le fractionnement azoté appliqué aux céréales immatures met en évidence des différences qualitatives importantes (tabl. 2). Comparé à celuii du maìs à la récolte, le taux d'azote soluble de la céréale est réduit sous l'effet de la déshydratation $(-46$

\section{TABLEAU 2}

Fractionnement azoté du maïs immature et de ses produits de conservation (en p. Ioo $\mathrm{N}$ total)

Nitrogen fractions of immature ear corn with husks

(p. Ioo total N)

\begin{tabular}{|c|c|c|c|}
\hline \multirow{2}{*}{$\begin{array}{l}\text { Fraction azotée } \\
\text { (Nitrogen fraction) }\end{array}$} & \multicolumn{3}{|c|}{$\begin{array}{l}\text { Épi de mais avec spathe } \\
\text { (Immature maize cob) }\end{array}$} \\
\hline & $\begin{array}{l}\text { A la récolte } \\
\text { (At harvest) }\end{array}$ & $\begin{array}{c}\text { Ensilé } \\
\text { (Silage) }\end{array}$ & $\begin{array}{c}\text { Déshydraté } \\
\text { (Dried) }\end{array}$ \\
\hline \multirow{2}{*}{\multicolumn{4}{|c|}{$\begin{array}{l}\mathrm{N} \text { soluble T.C.A. (T.C.A. } \\
\text { soluble } N)\end{array}$}} \\
\hline & $1^{\prime} \mathbf{k}, \mathbf{\prime}^{\prime}$ & 39,8 & 7,9 \\
\hline $\mathrm{N}-\mathrm{NH}_{3} \ldots \ldots \ldots \ldots$ & 一 & 4,0 & 一 \\
\hline $\begin{array}{l}\text { Albumine }+ \text { globuline } \\
\text { (Albumin }+ \text { globulin }) \ldots\end{array}$ & 4,3 & 2,1 & 2,0 \\
\hline Prolamine (Prolamin)..... & 17,6 & 10,6 & 16,9 \\
\hline N résiduel (Insoluble $N$ ) .. & $6+2$ & 47,2 & 73,2 \\
\hline
\end{tabular}

\section{TABLEAU 3}

Teneur en certains acides aminés de l'épi de maïs immatuve (g/16 g N) Amino acid content of immature ear corn with husks (g/I6 g N)

\begin{tabular}{|c|c|c|c|c|c|c|}
\hline \multirow{2}{*}{$\begin{array}{c}\begin{array}{c}\text { Épi de maïs } \\
(\text { Maize cob) }\end{array} \\
\text { Acides aminés }\end{array}$} & \multicolumn{2}{|c|}{$\begin{array}{l}\text { A Ia récolte } \\
\text { (At havvest) }\end{array}$} & \multicolumn{2}{|c|}{$\begin{array}{c}\text { Ensilé } \\
(\text { Silage })\end{array}$} & \multicolumn{2}{|c|}{$\begin{array}{c}\text { Déshydraté } \\
\text { (Dried) }\end{array}$} \\
\hline & $\begin{array}{c}\text { Total } \\
(\text { Total })\end{array}$ & $\begin{array}{l}\text { Libre } \\
\text { (Free) }\end{array}$ & $\begin{array}{c}\text { Total } \\
\text { (Total) }\end{array}$ & $\begin{array}{l}\text { Libre } \\
\text { (Free) }\end{array}$ & $\begin{array}{c}\text { Total } \\
(\text { Total })\end{array}$ & $\begin{array}{l}\text { Libre } \\
\text { (Free) }\end{array}$ \\
\hline Lysine $\ldots \ldots \ldots \ldots \ldots \ldots$ & 2,3 & 0,46 & 1,6 & 1,01 & 2,4 & 0,18 \\
\hline Arginine $\ldots \ldots \ldots \ldots \ldots \ldots$ & $t_{x}, 9$ & 0,27 & 3,4 & 0,12 & 4,8 & 0,09 \\
\hline Tyrosine $\ldots \ldots \ldots \ldots \ldots \ldots$ & 4,1 & 0,15 & 3,6 & 0,35 & 4,2 & 0 \\
\hline Proline $\ldots \ldots \ldots \ldots \ldots \ldots$ & 9,1 & 0,57 & 9,4 & 1,49 & 9,1 & 0,39 \\
\hline Ac. aspartique $\ldots \ldots \ldots \ldots \ldots$ & 6,9 & 0,65 & 6,4 & 1,47 & 6,8 & 0,32 \\
\hline Ac. glutamique $\ldots \ldots \ldots \ldots \ldots$ & 18,0 & 0,35 & 17,5 & 1,74 & 18,9 & 0,32 \\
\hline Glutamine + asparagine ..... & — & 0,31 & — & 0,82 & - & 0,15 \\
\hline Ornithine $\ldots \ldots \ldots \ldots \ldots \ldots$ & 一 & 1,58 & 一 & 2,85 & - & - \\
\hline
\end{tabular}


p. IOo) et très fortement accru par l'ensilage ( + I74 p. IOO), l'azote ammoniacal ne représentant qu'une faible part de cette augmentation. La fraction " albumines + globulines " diminue également dans les deux traitements, celle de la prolamine est surtout réduite dans l'ensilage. De l'hydrolyse partielle des protéines sous l'action fermentaire résulte une réduction sensible de la fraction d'azote résiduel dans l'ensilage.

Ia composition en acides aminés totaux (tabl. 3) n'est notablement modifiée que dans l'ensilage, où l'on note une diminution des taux de lysine ( -30 p. Ioo) d'arginine et de tyrosine et l'apparition d'ornithine. Les acides aminés libres s'y retrouvent pour environ 20 à $25 \mathrm{p}$. roo, sauf la lysine, la tyrosine et l'arginine dont la forme libre représente respectivement $42-2$ et 9 p. Ioo des teneurs du maïs initial. Ainsi, sous l'effet de ces transformations, 26 p. Ioo seulement de la lysine initiale est retrouvée incluse dans le résidu azoté insoluble de l'ensilage.

b) Glucides.

I.es taux d'amidon (35,6 p. Ioo) et de glucides alcoolosolubles ( I p. Ioo) dans la matière sèche de l'ensilage sont nettement inférieurs à ceux de l'épi de maìs déshydraté (respectivement 4I,6 et 8,4 p. I00) ; les fractions hydrosolubles diffèrent peu (respectivement 93 et 65 p.p.m.). L'amidon facilement attaquable passe de 20 p. Ioo à la récolte, à I6 p. Ioo dans l'ensilage et 48 p. Ioo dans le produit déshydraté.

\section{TABLEAU 4}

Résultats moyens journaliers par période et par animal

Daily individual average balance data

\begin{tabular}{|c|c|c|c|c|c|c|c|}
\hline \multirow{2}{*}{$\begin{array}{c}\text { Régime, animal } \\
\text { période } \\
\text { (Diet, animal } \\
\text { period) }\end{array}$} & \multirow{2}{*}{$\begin{array}{c}\text { Poicls vif } \\
(\text { Live } \\
\text { weight }) \\
(\mathrm{kg})\end{array}$} & \multirow{2}{*}{$\begin{array}{c}\text { Gain } \\
\text { de poids } \\
(\text { Ileight } \\
\text { gain) } \\
(\mathrm{k} \times / \mathrm{j})\end{array}$} & \multirow{2}{*}{$\begin{array}{c}\text { MS ingérée } \\
\text { (Dry matter } \\
\text { intake } \\
\left(g / \mathrm{kg} \mathrm{I}^{0,75}\right)\end{array}$} & \multicolumn{4}{|c|}{$\begin{array}{l}\text { Azote (g) } \\
\text { (Nitrogen) }\end{array}$} \\
\hline & & & & $\begin{array}{l}\text { Ingéré } \\
\text { (Intake) }\end{array}$ & $\begin{array}{c}\text { (rine } \\
\text { (Lrine) }\end{array}$ & $\begin{array}{c}\text { lèces } \\
\text { (Feces) }\end{array}$ & $\begin{array}{c}\text { Bilan } \\
\text { (Balance) }\end{array}$ \\
\hline E 12 I & $5: 3$ & 0,06 & 68,4 & 17,3 & 11,6 & 4,7 & 1,0 \\
\hline E $13 \mathrm{I}$ & 54,2 & $0,09 !$ & 63,9 & 16,3 & 8,9 & 5,1 & $2, ? 3$ \\
\hline E $5 \mathrm{II}$ & $81, k$ & 0,59 & 101,2 & 36,3 & 20,1 & 10,6 & 5,6 \\
\hline E 1'́t II & 62,1 & 0 & 67,5 & 19,3 & 9,9 & 5,5 & 3,9 \\
\hline ES $6 \mathrm{I}$ & $66 i, f$ & 0,71 & 95,9 & $5^{\prime}, 7$ & 26,9 & 10,8 & 17,0 \\
\hline ES $11 \mathrm{I}$ & 58,4 & 0,61 & 90,0 & 46,8 & 24,2 & 9,9 & 12,7 \\
\hline US: II & 75,0 & 0,19 & 85,7 & 52,3 & 28,8 & 11,4 & 12,1 \\
\hline ES $10 \mathrm{II}$ & 61,5 & $0,6 \geq$ & $9_{4}^{\prime}, 3$ & 49,8 & 20,2 & 9,6 & 18,0 \\
\hline $\mathrm{D} 4 \mathrm{I}$ & 64,8 & 0,36 & 82,1 & 26,3 & 11,7 & 11,6 & 3,2 \\
\hline D $10 \mathrm{I}$ & 53,0 & 0,11 & 73,0 & 20,1 & 9,1 & 0,2 & 1,8 \\
\hline 1) $6 \mathrm{II}$ & 76,8 & $0,3: 3$ & 92,8 & 32,3 & 13,8 & $1 / 1,0$ & 1,5 \\
\hline D $111 \mathrm{I}$ & 64,9 & $0,3: 2$ & 81,1 & 25,8 & 11,7 & 10,9 & 3,2 \\
\hline DS $5 \mathrm{I}$ & 69,4 & 0,74 & 95,7 & 57,9 & 24,3 & 13,0 & 20,6 \\
\hline ISS $1^{\prime}+1$ & 59,9 & 0,69 & 92,0 & 49,7 & 16,5 & 13,4 & 19,8 \\
\hline DS 12 II & $6 \%, 7$ & 0,60 & 89,3 & 50,0 & 20,2 & 12,5 & 17,0 \\
\hline DS $13 \mathrm{II}$ & 66,3 & 0,61 & 92,9 & 53,2 & 13,7 & 13,8 & 25,7 \\
\hline
\end{tabular}




\section{2. - Bilan digestif et métaboliquz}

Les résultats des bilans de matière sèche et d'azote sont regroupés dans les tableaux 4 et 5 . Le niveau d'ingestion de l'ensilage distribué seul est plus faible et surtout plus variable que celui du maïs séché; mais quelle que soit sa forme de présentation, l'épi de maïs distribué seul ne procure qu'une croissance limitée et un

\section{TABLEAU 5}

Utilisation digestive des composants du régime et bilan azoté

Digestibility of dietary components and nitrogen balance

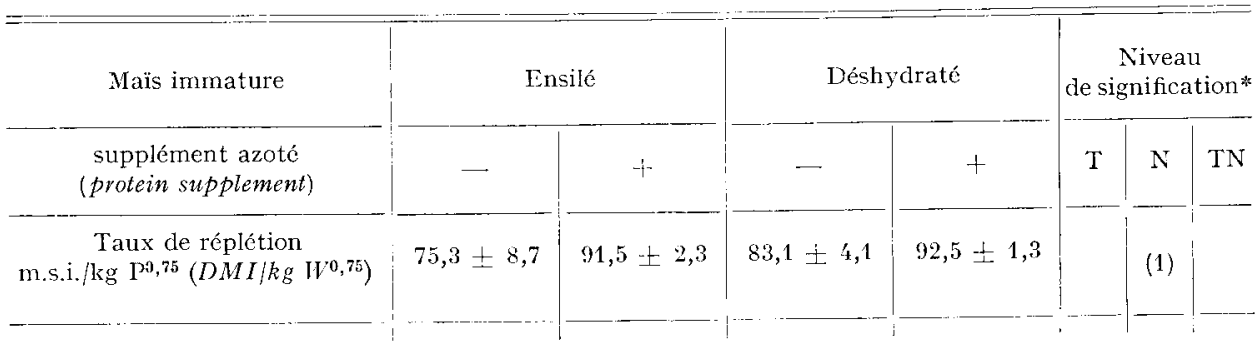

Utilisation digestive $(\% \pm \mathrm{sm})$ (Digestibility)

\begin{tabular}{|c|c|c|c|c|c|c|c|}
\hline Matière sèche (Dry matter) & $77,1 \pm 0,1$ & $74,8 \pm 0,8$ & $73,4 \pm 0,7$ & $72,2 \pm 0,7$ & (2) & (1) & \\
\hline Mat. organique (Organic matter) & $78,9 \pm 0,4$ & $76,7 \pm 0,7$ & $75,1 \pm 0,6$ & $73,9 \pm 0,7$ & $(2)$ & & \\
\hline Azote (Nitrogen) & $71,0 \pm 0,9$ & $79,5 \pm 0,6$ & $56,0 \pm 0,8$ & $7 \leftarrow, 7 \pm 1,0$ & $(2)$ & $(2)$ & (2) \\
\hline Cellulose brute (Crude fibre) & $4^{\prime}, 6 \pm 3,6$ & $34,8 \pm 6,4$ & $38,4 \pm 3,2$ & $26,8 \leq 2,4$ & & (1) & \\
\hline Mat. grasse brute (Crude fat) & 85,4 土 1,7 & $79,1 \pm 1,3$ & $68,4 \pm 0,7$ & $65,8 \pm 0,8$ & (2) & $(2)$ & \\
\hline $\begin{array}{l}\text { Extractif non azoté } \\
(N \text { free extract })\end{array}$ & $84,3 \pm 0,3$ & $81,7 \pm 0,6$ & $81,7 \pm 0,5$ & $80,5 \pm 0,4$ & (2) & (2) & \\
\hline
\end{tabular}

Bilan azoté ( $\pm \mathrm{sm})$ (Nitrogen balance)

\begin{tabular}{|c|c|c|c|c|c|c|c|}
\hline Bilan apparent $(g / j)(N$ balance $)$ & $3,2 \pm 1,0$ & $15,0 \pm 1,5$ & $3,1 \pm 0,6$ & $20,8 \pm 1,8$ & (1) & (2) & (1) \\
\hline $\begin{array}{c}\text { Coefficient d'utilisation } \\
\text { pratique }(\%) \\
(N \text { balance } p .100 \mathrm{~N} \text { intake) }\end{array}$ & $13,9 \pm 3,0$ & $29,4 \pm 2,8$ & $11,6 \pm 1,1$ & $39,5 \pm 3,2$ & & $(2)$ & \\
\hline $\begin{array}{l}\text { Coefficient de rétention ( } \%) \\
\text { (Apparent retention) }\end{array}$ & $19,6 \pm 4,2$ & $36,9 \pm 3,2$ & $20,6 \pm 1,9$ & $52,9 \pm 4,6$ & (1) & (2) & \\
\hline
\end{tabular}

* Source de variation : $\mathrm{T}$ : traitement (processing)

N : niveau azoté $\left(N^{+}\right.$level $)$

$\mathrm{TN}$ : interaction.

Niveau de signification (Level of significance)

(1) $0,01<P<0,05$

(2) $\quad \mathrm{P}<0,01$. 
bilan azoté faiblement positif. L'addition de tourteau accroît le niveau d'ingestion, qui devient comparable pour les deux régimes, et élève le bilan azoté, beaucoup plus nettement dans le cas de la céréale déshydratée. L'interaction entre les effets du traitement et du taux azoté du régime est, de ce fait, significative. Les coefficients de rétention ( $\mathrm{N}$ retenu $\mathrm{p}$. roo $\mathrm{N}$ digéré) et d'utilisation pratique ( $\mathrm{N}$ retenu $\mathrm{p}$. Ioo $\mathrm{N}$ ingéré) varient dans le même sens que les bilans.

L'utilisation digestive des composants principaux des régimes (tabl. 5) dépend, de manière très significative, sauf pour la cellulose brute, du mode de conservation, de la céréale ; l'ensilage est, à cet égard, supérieur à la déshydratation. Au niveau azoté élevé correspond, quel que soit le traitement, une meilleure utilisation digestive apparente de l'azote et une réduction de celle des autres composants vraisemblablement liée au niveau plus élevé d'ingestion de matière sèche par les animaux.

\section{TABLEAU 6}

Digestion de l'épi de maïs immature chez le porc: transit aqueux, amidon et activité $\alpha$-amylasique dans les contenus digestifs

Digestion of immature ear corn in the pig:

liquid flow, starch, $\alpha$-amylase activity in gut contents

\begin{tabular}{|c|c|c|c|c|c|}
\hline $\begin{array}{l}\text { Épi de maïs } \\
\text { (Earn corn) }\end{array}$ & \multicolumn{2}{|c|}{$\begin{array}{c}\text { Ensilé (E) } \\
\{\text { Ensiled }(E)\}\end{array}$} & \multicolumn{3}{|c|}{$\begin{array}{c}\text { Déshydraté (D) } \\
(\text { Dried }(D))\end{array}$} \\
\hline $\begin{array}{c}\text { Heures après } \\
\text { le repas } \\
\text { (Hours after meal) }\end{array}$ & 1 & 3 & $1 / 2$ & 1 & 3 \\
\hline
\end{tabular}

PEG (p. 100 du total retrouvé) (PEG (p. 100 of total recovered))

\begin{tabular}{r|r|r|r|r|c} 
Estomac (Stomach) & 38 & 36 & 26 & 21 & 19 \\
$\begin{array}{r}\text { Intestin grêle } \\
\text { (Small intestine) }\end{array}$ & 62 & 64 & 74 & 79 & 43 \\
Caccum (Caecum). & 0 & 0 & 0 & 0 & 12 \\
Gros intestin (Lar- & 0 & 0 & 0 & 0 & 26 \\
ge intestine)... & 0 & &
\end{tabular}

Amidon (p. 100 de l'ingéré) (Starch ( $p, 100$ of intake))

\begin{tabular}{l|c|c|c|c|c} 
Estomac (Stomach) & 26,4 & 37,8 & 55 & 64,5 & 52,5 \\
$\begin{array}{c}\text { Intestin grêle } \\
\text { (Small intestine) }\end{array}$ & 0,5 & 0,5 & 7,6 & 3,5 & 2,1 \\
$\begin{array}{c}\text { Cæcum (Caecum) } \\
\text { Gros intestin (Lar- } \\
\text { ge intestine ... }\end{array}$ & 0,4 & 0,1 & 1 & 1 & 0,3 \\
\hline
\end{tabular}

Activité amylasique (U. Somogyi/ml) (Amylase activity)

\begin{tabular}{r|r|r|r|r|r} 
Estomac (Stomach) & 25,5 & 23,8 & 5,8 & 2,5 & 8,2 \\
Intestin grêle & & & & & \\
$\quad$ (Small intestine) & 145 & 27 & 303 & 1400 & 460 \\
Cacum (Caecum). & 0 & 0 & 2,9 & 0 & 0 \\
Gros intestin (Lar- & & 0 & 0 & 0 & 0 \\
ge intestine)... & 0 & 0 & & \\
\hline
\end{tabular}




\section{3. - Analyse des contenus digestifs}

Nous avons déterminé les quantités de PEG, d'amidon, de glucides solubles et l'activité amylasique aux différents niveaux du tube digestif de porcs abattus après le repas test (tabl. 6).

a) Polyéthylène-glycol (PEG).

Le PEG, dont 82 à 100 p. Ioo de l'ingéré ont été récupérés, se répartit dans le tube digestif de manière différente selon le régime. Avec le déshydraté, ce traceur de la phase aqueuse quitte l'estomac très vite après le repas et, après la première demi-heure, il n'en reste dans cet organe que $26 \mathrm{p}$. Ioo. Par la suite, la vidange stomacale est plus lente, mais on constate qu'il reste beaucoup plus de PEG présent I et 3 heures après le repas dans l'estomac des animaux recevant l'ensilage ( 38 et $36 \mathrm{p}$. Ioo respectivement) que chez ceux qui consomment le maïs déshydraté (2I et I9 p. Ioo). Cet effet de passage plus rapide du PEG dans le tube digestif est également constaté dans les segments distaux : 3 heures après le repas, on trouve $\mathrm{I} 2$ et I6 p. Ioo du PEG dans le cæcum et le gros intestin de l'animal recevant le mais déshydraté, alors que ce traceur est absent des mêmes organes du porc nourri au maïs ensilé.

b) Quantité d'amidon et activité amylasique.

Au niveau stomacal, on trouve beaucoup plus d'amidon avec le régime D (55 p. Ioo de l'ingéré) qu'avec le régime $\mathrm{E}$ ( $30 \mathrm{p}$. IOO) ; ces taux n'évoluent guère durant les trois heures qui suivent le repas. L'activité amylasique est importante après ingestion de 1'ensilage.

Au niveau intestinal, la quantité d'amidon provenant du maïs déshydraté décroît lentement après le repas ; elle reste toujours très supérieure à celle de cette même substance dans les contenus digestifs des animaux à l'ensilage. L'activité amylasique est, entre I heure et 3 heures après ingestion d'ensilage, nettement inférieure à celle observée aux mêmes temps après le repas de maïs déshydraté.

Dans le cæeum et le gros intestin, on ne retrouve d'amidon que chez les animaux recevant du maïs déshydraté; l'activité amylasique de la phase liquide séparée par centrifugation reste dans ces organes très faible quel que soit le régime.

c) Quantité et nature des glucides solubles.

Les glucides hydrosolubles précipitables à l'iode sont présents dans les aliments à très faible dose (D : $65 \mathrm{mg} / \mathrm{kg} \mathrm{MS} ; \mathrm{E}: 93 \mathrm{mg} / \mathrm{kg} \mathrm{MS})$. On en retrouve des teneurs comparables dans les contenus stomacaux.

La concentration stomacale des glucides alcoolosolubles est pratiquement identique à celle des aliments après une heure $(8,0 \mathrm{p}$. Ioo pour $1 \mathrm{le} \mathrm{D}, \mathrm{I}, 4 \mathrm{p}$. Ioo pour E). Eille est peu modifiée après 3 heures ( 14 contre Io $\mathrm{mg} / \mathrm{g}$ ). Dans l'estomac des animaux abattus, la présence de maltose et de maltotriose est constante. Le saccharose et le raffinose, encore présents, 3 heures après le repas, à l'état de traces dans les contenus stomacaux de porcs aut régime $\mathrm{D}$, sont absents chez les animaux nourris à l'ensilage. Le glucose n'est présent, en faible quantité qu'avec le régime $\mathrm{D}$, une heure après le repas.

Dans l'intestin grêle, les concentrations en glucides alcoolosolubles sont élevées ; elles restent constantes durant la digestion du mais déshydraté ( $11_{5} \mathrm{mg} / \mathrm{g} \mathrm{MS}$ ) alors 
qu'avec l'ensilage, elles diminuent (resp. 244 et $\mathrm{I}_{57} \mathrm{mg} / \mathrm{g}$ MS, I et 3 heures après le repas). Dans les deux cas, les glucides présents vont du glucose au maltopentaose avec de très faibles concentrations de maltotétraose. I) ans le cæcum et le gros intestin, les quantités de glucides alcoolosolubles sont faibles et de longueut de chaîne plus élevée.

\section{DISCUSSION}

Comme le montrent clairement les résultats de bilan et d'abattage, le mode de conservation de l'épi de maïs immature influence de façon déterminante l'utilisation digestive et métabolique de l'azote et de l'énergie du régime.

L'utilisation digestive apparente de l'azote de l'épi de mais est meilleure avec l'ensilage qu'après déshydratation, effet déjà noté avec le maïs mûr chez le porcelet par Fevrier, Aumaitre et Salmon-LEGagneur (197I). La différence observée peut être due soit à un chauffage excessif lors du séchage de l'épi, soit à la faible durée de la période d'adaptation des animaux.

Si, à ces deux régimes, nous appliquons la correction d'azote métabolique proposée par ZELTER et CHARLET-LERY (I96I), qui correspond à I,2 p. IOO de la matière sèche fécale, la digestibilité réelle de l'azote de la céréale s'élève à $9 \mathrm{I}, 9$ pour l'ensilage et à 79,5 avec l'épi séché : le traitement thermique paraît donc bien la réduire, ce que laissait prévoir l'accroissement de la fraction d'azote insoluble dans le produit déshydraté. Dans l'ensilage par contre, la composition de la fraction azotée soluble est modifiée; la teneur de l'épi en azote ammoniacal et en certains acides aminés libres est fortement accrue. L'importance des actions fermentaires sur l'azote de la céréale, dont l'évolution de la teneur en lysine est un bon indice, explique l'égalité des bilans azotés observée après ingestion de la céréale seule, malgré la meilleur utilisation de l'ensilage; mais elle ne suffit pas à justifier à elle seule l'efficacité relative plus grande de l'azote du régime déshydraté supplémenté par le tourteau de soja.

En ce qui concerne les glucides, un contraste net est établi entre la digestion rapide et précoce de l'amidon de l'ensilage dans l'estomac, où règne une forte activité amylasique, provenant pour environ un tiers de l'ensilage même, et la dégradation tardive de l'amidon du produit déshydraté, présent dans le cæecum trois heures après le repas. Il apparaît ainsi que ni la quantité totale des glucides, ni le moment de leur hydrolyse dans la lumière intestinale ni donc la nature des métabolites qui en résultent sous l'influence conjuguée des enzymes digestives et de la flore microbienne ne sont comparables dans les deux régimes ; cette disponibilité différente de l'énergie constitue un élément d'interprétation du meilleur bilan azoté que procure la céréale déshydratée complétée par un apport protéique équilibré. Nous avons également constaté la vitesse de transit plus rapide de l'épi immature déshydraté, qu'atteste l'évolution des quantités de PEG et de cellulose retrouvées dans les compartiments du tube digestif. L'acidité du régime pourrait en être la cause : en effet, si, chez le porc en croissance, AUFFray, MarTinét et RÉRAT (I967) ne constatent qu'un effet de faible ampleur de l'acidité en régimes purifiés contenant une solution acide tamponnée, avec du maïs acidifié, HoLMES, BAYLEY et HoRNEY (1974) notent par contre un net ralentissement de la vidange gastrique, phénomène qu'observent également HunT et KNOX (I972) chez l'Homme par adjonction d'acide au repas. 
Il est par ailleurs intéressant de noter que l'amiclon de maïs ensilé est plus complètement dégradé in vivo que celui du déshydraté, alors que ce dernier est beaucoup moins résistant à l'amylase bactérienne in vitro: bien que la différence ne porte que sur une faible fraction de l'amidon ingéré, notre observation peut être rapprochée de celle de Szylit, DilorT-I,Aval et Borgida (I974). Ces auteurs mettent en évidence que l'action de deux amylases, l'une bactérienne, l'autre pancréatique agissant ensemble ou séparément, en quantités isoactives, sur des amidons de référence, conduit à des maltooligoholosides de tailles différentes. Aux lieux et aux temps variables où l'amidon des régimes est attaqué, pourraient correspondre des mélanges d'amylases qui conduisent à la formation d'oligoholosides différents; leur présence pourrait expliquer les discordances entre test enzymatique et effet biologique.

Sur le plan pratique, l'épi de maîs, qu'il soit conservé par ensilage ou séchage artificiel, ne peut satisfaire que les besoins azotés d'entretien du porc en finition. Un supplément azoté équilibré, qui double la teneur protéique du régime, assure une croissance correcte de l'animal recevant la céréale déshydratée. Ce supplément doit être accru d'environ un tiers, si l'on veut obtenir des animaux une performance de croissance équivalente, à partir de l'épi de mais ensilé. La teneur élevée en cellulose de ces régimes est susceptible de conduire, même en alimentation à volonté, à la production de carcasses de faible adiposité (FEvrier, AumaITre et SALMON-LEGAGNEUr, I97I). La plus grande digestibilité des composants du maîs ensilé lui confère une valeur énergétique nette supérieure à celle du produit déshydraté (resp. 0,88 contre $0,8 \mathrm{I} \mathrm{UF} / \mathrm{kg}$ de matière sèche). Cette différence compense les pertes plus élevées de l'ensilage qu'une technologie adaptée est capable de maintenir dans des limites acceptables (Gouet, Riou et Bousset-Fatianoff, r97I). Dans la mesure où seront disponibles des chaînes adaptées de récolte du maïs et de distribution de l'ensilage à l'auge après incorporation des compléments utiles, l'épi de maïs ensilé sera susceptible de fournir une part non négligeable du régime du porc en finition dans les zones d'élevage oì cette culture est maintenant établie.

$$
\text { Rę̧u pow publication on mars } 19 \% 5 .
$$

\section{SUMMARY}

EFFECTS OF STORAGE TECHNIQUE FOR IMMATURE MAIZE

ON THE UTILIZATION OF ITS CARBOHYDRATES AND NITROGEN

IN GROWING-FINISHING PIGS

Immature (50 p. I oo DM) ear corn chops with husks were processed either by a I $30^{\circ} \mathrm{C}$, I hour air drying or by ensiling. The effect of these treatments on the composition of the products and their digestibility and protein efficiency in the growing pig were compared :

1) Drying slightly reduced prolamine $\mathrm{N}$ ( 17.6 to $\mathrm{i} 6.9 \mathrm{p}$. 1oo total $\mathrm{N}$ ) and albumin + globulin $\mathrm{N}(4.3$ to $2.0 \mathrm{p}$. IOO N) and increased in vitro the starch degradation by $B$. subtilis alpha-amylase (table 2).

Ensiling increased TCA soluble N ( $\mathrm{I}_{4} .5$ to $39.8 \mathrm{p}$. Ioo total $\mathrm{N}$ ) and ammonia N (4 p. Ioo total $\mathrm{N}$ ) ; some amino acids were reduced (e. g. lysine : - 30 p. Ioo). Soluble carbohydrate and starch were partly fermented into lactic acid and volatile fatty acids (table 3 ).

2) The silage components were significantly $(\mathrm{P}<0 . \mathrm{I})$ better digested by the finishing 
swine than those of the dried ear corn especially for protein ( 7 1.0 against 56.0 for clry and ensiled corn).

3) Soybean meal + DL-methionine supplementation in an adequate amount (I6 p. Ioo of DM intake), increased the nitrogen retention with the dried cereal. Interaction between processing and protein level was significant (table 5).

4) On 5 barrows slaughtered 30,60 or $180 \mathrm{mn}$ after a test meal, with PEG as a marker, an increased feed passage and a delayed starch digestion was shown with the dried ear corn. With the silage, a higher alpha-amylase activity (table 6) and a slower emptying of the stomach improved the disappearance of starch from that organ.

\section{RÉFÉRENCES BIBLIOGRAPHIQUES}

Auhray P., Martinet J., Rérat A., ig67. Quelques aspects du transit gastro-intestinal chez le Porc. Ann. Biol. anim. Bioch. Biophys., 7, 26r-679.

Barnett A. J. Cx., I951. The colorimetric determination of lactic acid in silage. Biochem. J., 49,527-528.

Baudet J., Mosse J., Landry J., Motrkaux T., 1966. Étude sur les protéines dumaïs I. Composition en acides aminés des fractions azotées du grain. Ann. Physiol. veg., 8, 32I-329.

Colombus A., Harvisch W., Jand S., I957. Untersuchungen über den Futterwert von Grün-und Silomaïs beim Einsatz an Wiedcrkaüer und Schweine. Arch. Tisvernährung, 7, I34-2II.

Commerford J. D., Van Duzee Cr. T., Scaller B. L., 1963. Macropaper chromatograph of starch hydrolysate. Cereal Chem., 40, $482-486$.

Conway E. J., I957. Microdiffusion analysis and volumetric errors, $4^{\mathrm{c}}$ ed., Crosbr, Lockwood and Son, London, $465 \mathrm{p}$.

Farries F. E., Ostage, H. J., I96r. Zur Technik langfristiger Stoffwechselversuche an wachsenden Schweinen. Z. Tierphysiol. Tiercruähr. Futtermittelk., 16, I I-z9.

Fatianorf N., Gouet P., r969. Relation permettant de corriger rapidenent et avec précision la matière sèche des ensilages séchés à l'étuve. Ann. Zootech., 18, 407-418.

Fevrier C., Aumaitre A., Salmon-Legagneur İ., ig7r. Valeur alimentaire du maïs ensilé à différents stades de maturité pour la truic, le porcelet, le porc en croissance-finition. J. Rech. Porc. France, I37-148., I'aris, I. N. R. A., I. T. 1'. ed.

Govet P., Riou Y., Bovssit-Fatianof N., ig7r. Conservation par ensilage ou déshydratation d'une orge et d'un nais immatures. Ann. Zoolech., 20, 275-290.

Giri K. V., Nigam V. N., I953. Separation of simple obligosaccharides by circular paper chromatography. Naturwiss, 40, 343-344.

Gross F., I97o. Schlussfolgerungen für den Maïszüchter aus den Gruber Fütterungsversuehen mit Silo und Körnermaïs. Bayer. landn. Jahrbuch, 47, 235-240.

Hassid W. Z., Netreld K. F., 1968. Quantitative determination of starch in plant tissues. In Carbohydrate Chemistry, R. L. Winstler, vol IV, 33-36, Acad. Press., Londres.

Heliberg A., I962. Spannmalsensilernung är enkel och sächer. Lantmannen, 36, 823-825.

Holmes J. H. G., BAYLEY H. S., Iorney IF. D., I973. Digestion and absorption of dry and high moisture maize diets in small and large intestine of the pig. Br.J. Nutr., 30, 40I-4Io.

Hotmes J. H. G., Bayley H. S., Horney F. I)., 1974. Digestion of dry and high moisture maize diets in the stomach of the pig. Br. J. Nutr., 32, 639-646.

Huber C. N., Scobell H., Hax Tai., I966. Determination of corn syrup by direct densitometry of thin layer chromatography. Cereal Chem., 43, 342-346.

Hunr J. N., KNox M. T., 1972, The slowing of gastric emptying by four strong acids and three weak acids. J. Physiol., London, 222, I87-208.

KESLER R. B., Ig66. Ion exchange chromatography of sugar wood puip and paper hydrolysates. In Automation in Analytical Chemistry. 174-177, Nediad inc., New-York.

Mercifr C., 1968. Contribution à l'étude de la structure du grain d'amidon au moyen de méthodes physiques et enzymatiques. Thèse Doc. État. No C. N. R. S. AD 2413.

Moore S., Spackmaxis D. H., Strin IV. M., r958. Chromatography of amino acidis on sulfonated polystyrene resins. Anal. Chem., 3, I I85-I igo.

Sмiтн R. H., r959. The development and function of rumen in mills fed calves. J.agric. Sci., 52, 72-78.

Szylit O., Delort-laval J., Borgida L. P., I974. Dégradation dans le jabot du Coq et efficacité d'amidons de mais à différents taux d'amylose sur la croissance du Poulct. Ann. Zootech., 23, $253^{-265}$.

Thivend P., Mercier C., Guilbot A., 1965. Dosage de l'amidon dans les milieux complexes. Ann. Biol. anim. Bioch. Biophys., 5, 5 I 3-536.

Zelter S. Z., Charlet-Lery G., $196 \mathrm{I}$. Lifficacité de quelques protides alimentaires chez le porc. I. Ann. Biol. anim. Bioch. Biophys., 1, 29-46. 
Zelter S. Z., Charlet-Lery G., Tisserand J. L., I97i. Influence chez le Taurillon en croissance, du traitement de conservation (ensilage ou déshydratation) de la céréale immature (orge, mais) sur sa valeur nutritive et sur l'efficacité métabolique de l'urée ajoutée. Ann. Zootech., 20, I35-I52.

Zelter S. Z., Leroy F., I958. Azote uréique et activité bactérienne in vitro au niveau du rumen. Ann. Zootech., 7, I73-183.

Anonyme, Ig68. The determination of amylase in serum, urine and other fluids, Tech. Bull., $\mathrm{n}^{\circ} 700$, SIGMA St Louis, Mo. 